\title{
APROXIMACIÓN TEÓRICA AL EFECTO PRODUCTO DEL ENCADENAMIENTO INSEGURIDAD PERSONAL - DESCONFIANZA GENERALIZADA - DIFERENCIAS DELICTIVAS COMUNITARIAS
}

\section{Theoretical approach to the product effect of chain-linking personal insecurity - widespread mistrust - community crime differencec \\ Jonathan Decle Castro ${ }^{1 *}$ \\ jonathandecle@hotmail.es

\author{
Marcos Pablo Moloeznik ${ }^{2 * \star}$ \\ mmoloeznik@yahoo.es
}

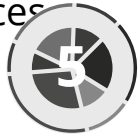

\section{Forma sugerida de citar:}

Decle Castro, Jonathan [et. al.]< (2016). Aproximación teórica al efecto producto del encadenamiento inseguridad personal-desconfianza generalizada-diferencias delictivas comunitarias. Revista Economía y Política, Año XII, No. 24. pp. 117-146

Fecha de recepción: 1 de diciembre de 2016

Fecha de aceptación: 30 de marzo de 2017

\section{Resumen}

El presente artículo discute y propone un vínculo teórico entre la inseguridad personal, la desconfianza generalizada y las diferencias delictivas comunitarias. De ahí que se divida en dos secciones: en la primera, la introducción y el marco teórico y analítico, se presentan los conceptos sociológicos y politológicos a los que se recurre, su alcance e interconexión. En la segunda, se expone la idea central de esta contribución: la eventual existencia de un fenómeno teórico funcional denominado efecto inseguridad personal -desconfianza generalizada-diferencias delictivas comunitarias. Finalmente, se ofrecen unas conclusiones.

PALABRAS CLAVE: inseguridad personal, confianza generalizada, instituciones, crimen.

\section{ABSTRACT}

This article discusses and proposes a theoretical link between personal insecurity, generalized mistrust and community crime differences. Hence it is divided into two sections: in the first, the introduction and the theoretical and analytical framework, the sociological and political concepts that are used, their scope and interconnection are presented. In the second, the central idea of this contribution is exposed: the possible existence of a theoretical functional phenomenon called personal insecurity effect-generalized mistrust-community delinquency. Finally, some conclusions are offered.

KEYWORDS: Personal insecurity, generalized trust, institutions, crime.

JEL: H56, K42

$1 *$ Doctor en Ciencias Económico Administrativas/Políticas Públicas por la Universidad de Guadalajara (México); Profesor de posgrados en la Universidad Juárez Autónoma de Tabasco (México).

2 ** Profesor-Investigador Titular del Departamento de Estudios Políticos, Centro Universitario de Ciencias Sociales y Humanidades de la Universidad de Guadalajara (México); Investigador Nacional Nivel II, Sistema Nacional de Investigadores. 


\section{A manera de introducción}

¿Qué hace que en un lugar se cometan más actos ilícitos que en otro? O bien, ¿por qué en una comunidad hay personas más propensas al delito que en otra? Esta es sin duda uno de los interrogantes más apasionantes como primigenios de la ciencia social. No obstante, la primera aproximación respaldada por una amplia batería de evidencia empírica de esta cuestión se remonta a la Escuela de Chicago (Harbeck, 2009); misma que deriva de los trabajos de Park (1925), Trasher (1927) y Shaw \& McKay (1942), y que enfatiza que el crimen es resultado de la fractura de las normas y la cohesión social (Bouffard \& Muftic, 2006). Esto es que la inestabilidad residencial (deterioro de los vínculos vecinales), la disfunción familiar (conflicto de valores, abandono y desapego de padres e hijos), la heterogeneidad étnica (conflicto entre personas con divergencia de valores y cultura), la pobreza, la desigualdad y el hacinamiento, son ingredientes que -mezclados en un contexto y espacio específico-, conllevan a distintos niveles de crimen entre las comunidades.

Posteriormente de dicho enfoque teórico se desprenden la anomia, y como una variante de la misma, la tensión. La primera arguye, en alusión a Durkheim (1897), que en medio de un cambio brusco de la estructura social, muchas veces autoritario, los individuos se ven conminados a romper las reglas y salirse del orden establecido (Rock, 2012). Empero en un segundo momento señala que el conflicto que se suscita entre las "metas ideales" que la sociedad enmarca como fines últimos de realización y los limitados recursos con los que cuenta el individuo para alcanzarlos (muchas veces un estado de pobreza y desigualdad que le producen "envidia"), es lo que lo motiva y da origen al delito (Merton, 1968; 1995). La tensión, por su parte, toma cierta distancia de la anomia que conlleva al "crimen por fallo estructural", al atribuirle orígenes más emocionales. Es decir, el acto de cometer un crimen es simplemente una reacción del individuo ante la desatención o el rechazo por parte de los otros (Agnew, 1992). Sin embargo, resulta notable que ambas teorías no son excluyentes entre sí, sino que más bien parecieran ser distintas dimensiones analíticas de un mismo fenómeno.

Por otro lado, llama igualmente la atención que todas estas causales del crimen parecieran responder a la necesidad inherente del hombre de "estar o sentirse seguro". En otras palabras, si bien la seguridad en el contexto cotidiano puede aludir a diversos encuadres u orientaciones, si se revisa con detalle, cualquier discusión sobre la misma partiría de la necesidad de resguardar los valores que más se aprecian y de poder contar con la capacidad de hacer elecciones (Williams, 2013). Lo que significa, en el sentido más elemental, el mantenimiento de la vida y "el poder hacer" (Ibidem). En efecto, esto se traduciría en la inexistencia de desigualdades económicas, políticas y sociales, tales como 
el desempleo, la pobreza, la marginación, la discriminación, la desunión familiar y étnica. (PNUD, 2014).

Dicho de otro modo, los individuos que comenten delitos por anomia, tensión y desorganización social (ATD), carecerían en el fondo de la seguridad. O bien, se sentirían inseguros respecto al referido problema de "sobrevivir y el poder hacer" y por ello delinquen. De ahí que, para apaciguar dicha inseguridad, el hombre ha creado tecnologías sociales que le generen estabilidad y certeza en su quehacer cotidiano, denominadas instituciones (North, 1990; Nelson \& Winter, 2002). Las hay formales, como las leyes y los reglamentos, la economía, el gobierno y el Estado; e, informales, como la familia, la historia y la cultura (North, 1990). Y son precisamente dichas fuentes institucionales los pilares de la confianza generalizada (Stolle, 2002); misma que a su vez se define como el grado con el que los individuos "perciben contar" o se "inclinan a cooperar" con los desconocidos (Stolle, 2002; Kouvo, 2011). Lo que dejaría entrever una posible relación funcional: entorno depresivo - proceso individual (luego compartido) vinculado con la noción de "estar o sentirse (in) seguro" - propensión delictiva por ATD-diferencias delictivas comunitarias.

De esta manera, la revisión de la literatura inserta la noción de que se estaría hablando de una elipse de conceptos que gravitan alrededor de una misma trama. Es decir, la propensión al delito por ATD acontecería por las mermas del entorno, pasaría por el marco de las instituciones que pretenden proveer seguridad pero que no lo hacen eficientemente; lo que a su vez, origina un proceso cognitivo individual compartido "por muchos" asociado con dicha inseguridad, que finalmente incide en las diferencias delictivas comunitarias. Por ende, tanto la intención criminal como las diferencias delictivas se interconectarían teóricamente con algún tipo de desconfianza micro o individual que es compartida - y que a la postre, derivaría en una consecuencia macro o generalizada específica.

\section{Marco teórico y analítico}

Hablar de forma sintética y conexa de la noción de seguridad, propensión al delito por ATD, institución y confianza, no es una tarea fácil. $Y$ no lo es no sólo porque al interior de dicho conceptos convergen diversos y extensos enfoques, sino porque cada una de estas perspectivas obliga a encontrar la orientación óptima que posibilite explicativamente el inferido nexo. De ahí la necesidad de abordar conceptos fundamentales, tales como seguridad, propensión al delito por ATD, instituciones, confianza, confiabilidad y confianza generalizada, que resultan apropiados para este propósito explicativo. 


\subsection{Seguridad}

La seguridad es imprescindible. Cada día las personas son transgredidas, desplazadas, golpeadas, secuestradas, torturadas, violadas, asaltadas, asesinadas y negadas a la alimentación, educación, empleo, sanidad y bienestar más elemental (Williams, 2013). No obstante, su concepto es subjetivo, elástico, contestatario y derivativo (Ibidem). Esto significa que no existe un consenso real sobre su significado, sino que más bien este depende de las circunstancias espaciales, temporales y coyunturales que la rodean. De ahí que no sea casual que la seguridad tenga tantas definiciones como ideologías, organismos gubernamentales e instituciones se puedan concebir. Y todavía más, la seguridad puede abordarse desde las diferentes esferas del saber, desde ciencias próximas tales como el derecho y la economía, hasta otras en apariencia distantes como la biotecnología y la informática. Así, se pueden mencionar la seguridad jurídica, la seguridad social, la seguridad alimentaria, la seguridad ambiental y la seguridad industrial, entre otras. $Y$ se consideran de igual manera la seguridad del individuo, de la sociedad y del mundo.

Lo anterior es un claro indicador de que la seguridad tiene distintas dimensiones. En este sentido, Williams (2013) propone acotar el análisis en función de qué es y de quién se trata cuando se habla de la misma. De este modo, define a la seguridad como la protección de los valores más preciados, lo que involucra contar con los medios necesarios para la sobrevivencia y el poder hacer elecciones; o bien, una "sobrevivencia plus" (Williams, 2013: 6). No muy lejos de esta posición se encuentra Bilgin (2013: 104), que la precisa como un estado ausente de amenazas tales como el dolor, el miedo, el hambre y la pobreza, cada uno de estos, elementos de la emancipación. Y es esta idea de la seguridad como emancipación la que explora Booth (1991: 319) al mencionar que "la seguridad significa ausencia de amenazas. La emancipación, por otro lado, refiere a la libertad de los individuos, o grupos, de aquellas restricciones que no permiten que puedan optar por las elecciones que ellos quieren hacer. La emancipación y la seguridad son, por tanto, dos caras de la misma moneda". Y concluye: "la emancipación, y no el poder u orden, es lo que produce verdadera seguridad".

Pero, ¿por qué ligar la seguridad con el poder y el orden? En efecto, ello alude a las dos visiones contrapuestas que, según Williams (2013), mayormente destacan en la seguridad. La acumulación de poder -es decir, se está seguro cuando se tienen suficientes recursos, propiedades, territorios, armas y dinero-, por un lado, versus el mencionado sentido emancipador de la seguridad. Esto es, se está seguro cuando se 
goza de justicia y derechos, en el entendido de que bajo esta óptica la seguridad no es más que un acuerdo entre actores (Williams, 2013). Así, el concepto de la seguridad gravita alrededor de estas tres consideraciones: su naturaleza derivativa, su rudimento contestatario y su arquetipo emancipador. De ahí que, mientas Beltrame (2011, p. 103) la entienda como "una fabricación de hechos que provienen de una visión mediática" y Collins (2013) como algo que está en constante debate, Williams (2013) la enfatice como una situación en la que no se tienen amenazas ni carencias.

No obstante, cuando se quiere comprender la seguridad de qué o quién está en juego, nuevamente Williams (2013) destaca que su primer objeto es el ser humano mismo; al tiempo que advierte que su discurso dominante ha sido durante décadas la protección del Estado. Se trata de una posición con la que concuerda Mutimer (2013), toda vez que la concibe como la estricta acción de resguardar a dicha institución de toda amenaza o riesgo externo e interno. Mientras que un tercer y cuarto enfoque son los grupos e identidades colectivas: burocracias, sociedades y regiones; y su orientación ecológica (Williams, 2013). Ésta última, entendiendo que "dado que los humanos son parte de la naturaleza y dependen de los sistemas ecológicos, sin un ambiente habitable, la discusión de todo lo demás se torna irrelevante" (Williams, 2013, p. 8). En síntesis, se estudia la seguridad de los individuos, de los grupos, de las sociedades y de los sistemas ambientales; empero, cualquier postura sobre la misma, parte de una base que garantizaría los medios necesarios para sobrevivir y el poder hacer elecciones (Williams, 2013).

\subsection{Propensión al delito por ATD}

El constructo teórico del crimen por anomia, tensión y desorganización social (ATD) se funda en la noción de que los factores del entorno incentivan, promueven e inducen al delito. Así, la primera de éstas, la anomia, teoría formulada por Merton (1968), delinea que el individuo es conminado al crimen porque no cuenta con los recursos o habilidades necesarias para alcanzar las metas que la sociedad le demanda como ideales de realización. Dicho en otras palabras, es un conflicto entre fines y medios (Merton, 1968). De ahí que, desde este enfoque, la pobreza, la desigualdad y el "efecto envidia" sean los condicionales de dicha intención (Kelly, 2000; Deller \& Deller, 2010). Entretanto que la tensión de Agnew (1992), variante que le atribuye orígenes más emocionales que estructurales a las causales del delito, argumenta que cuando las personas son desatendidas, rechazadas o ignoradas de algún modo, optarán por el crimen como reacción. 
Ahora bien, desde una perspectiva más amplia, la teoría de la desorganización social compara y explica los factores que generan diferencias delictivas entre comunidades (Harbeck, 2009). Así, derivado de los trabajos de Park (1925), Trasher (1927) y Shaw \& McKay (1942), Bouffard \& Muftic (2006) establecen que la inestabilidad y movilidad residencial, la ruptura familiar, la pobreza, la desigualdad, la heterogeneidad étnica y la densidad poblacional, son causales de crimen. Rock (2012), por su parte, señala que este enfoque centra su atención en la manera en cómo estos elementos interactúan en un determinado contexto espacial y cómo sus residentes reaccionan ante dichas situaciones. Sin embargo, puede observarse que cada uno de estos encuadres alude a distintas dimensiones de una misma lógica. Es decir, un individuo en una posición social desventajosa -de pobreza y desigualdad, por ejemplo- puede explicar su tendencia criminal a la vez tanto por no contar con los medios para realizarse, por percibir rechazo por parte de "los otros"; o bien, por ser parte de una comunidad deprimida socialmente. De ahí que se hable del crimen por ATD.

\subsection{Instituciones}

Las instituciones son el modo en como las cosas se hacen (North, 1990). Son patrones de conducta por los cuales optan los individuos toda vez que provienen de una racionalidad utilitaria. Dicho en otros términos, las instituciones son un medio para alcanzar un fin, ya sea instrumental o normativo. Tal diferencia queda expuesta tanto en su vertiente racional como organizacional (Campbell, 2009). Es decir, para la primera las instituciones son las reglas del juego que unos imponen para asegurar sus intereses u obtener ventajas sobre otros (North, 1990; North, 2005; Campbell, 2009). De ahí que éstas "sean producto de la lucha y la negociación" (Campbell, 2009, p. 11). Mientras que para la segunda, las instituciones son el resultado de una interactiva adaptación de individuos a los que el discurso colectivo les indica qué es lo apropiado (Scott \& Meyer, 1994; Scott, 2001; Campbell, 2009). Cualquiera sea la vertiente, en esencia las instituciones son mecanismos socio-cognitivos direccionados. 0 bien, artificios sociales que posibilitan el logro de objetivos (Nelson \& Winter, 2002), uno de los cuales, es la coordinación de esfuerzos conjuntos (Ostrom, 1990). En esto reside, justamente, la diferencia entre su fin "utilitario egoísta" y su fin "utilitario conveniente" (March \& Olsen, 1984; Campbell, 2009). 
Tabla 1.3.1 Orientaciones genéricas de análisis institucional

\begin{tabular}{|l|c|c|}
\hline \multicolumn{3}{|c|}{ Perspectivas de análisis institucional } \\
\hline Elección racional & Organizacional & Histórica \\
\hline
\end{tabular}

FUENTE: Elaboración propia con datos de Campbell (2009)

Así las instituciones limitan las alternativas puesto que restringen el rango de posibilidades de acción (North, 1990; North, 2005); inciden en el poder y la toma de decisiones, dado que discriminan la información y los recursos (Sharpf, 1997; Rutherford, 1994); e impactan positiva y negativamente en la capacidad de hacer innovaciones (Fligstein, 2001). De hecho, el estudio del cambio institucional, en el cómo y por qué las cosas comienzan a hacerse de distinta manera, hace hincapié en otra forma de abordarlas: su historicidad. La historia comparada de las sociedades describe por qué en unas y otras suceden distintas conformaciones de la estructura social; y por qué en unas y otras incluso la adopción de las mismas políticas conlleva a resultados divergentes (Thelen \& Steinmo, 1992). Más aún, esclarece quiénes fueron los actores que provocaron dichas variaciones, cómo las hicieron y qué circunstancias les permitieron realizarlas (Pierson, 2000).

En este sentido, Laso (2007) da cuenta que la institución bien puede entenderse como un entramado de procederes que puede variar inter-generacionalmente; y que su esencia gravita alrededor de la estabilidad y el cambio, y de la prueba y el error. En efecto, esto nuevamente la supone más como un instrumento que un fin en sí mismo, y más como un entramado racional - cognitivo interesado en la posibilidad de no perder o fracasar que en la de innovar o modificar. La innovación sería más bien una consecuencia de la búsqueda del éxito. En otras palabras, la institución se concibe como una estrategia óptima toda vez que funciona y disminuye el riesgo; o bien, como el camino ya probado. Es precisamente esta idea de seguridad la que enfatiza Laso por encima de otras perspectivas de la institución: la institución se consolida o no, en la medida en que se percibe segura.

\subsection{Confianza, confiabilidad y confianza generalizada}

La confianza es el determinante de la acción colectiva (Fukuyama, 1995; Lubell, 2007; Gambeta, 2008; Kramer, 2012). Es el fundamento en la construcción de redes e instituciones (Ostrom \& Ahn, 2007). Provee de certidumbre, posibilita la cooperación 
y la comunicación y coordinación entre individuos (Putman, 1993; Sonderskov, 2009; Padmanabhan, 2008). Su naturaleza descansa tanto en evaluaciones hipotéticas como en estimaciones futuras del comportamiento de otros (Gambeta, 2008). Y de suponer, que éstas (nos) serán apropiadas (Misztal, 1996). La confianza es también el vínculo entre sujetos que comparten creencias, identidades y valores (Adler-Lomnitz, 2012), por lo que se perciben iguales y predecibles (Ostrom \& Ahn, 2007; Adler-Lomnitz, 2012). Da sentido de estabilidad, al procurar el cumplimiento de reglas, normas, rutinas y hábitos (Robert, Dennis \& Hung, 2009); o bien, para la actuación segura de los individuos (Putnam, 1993; Kahan, 2003; Ostrom \& Ahn, 2007).

Otra consideración es que confiar involucra algún tipo de riesgo (Baier, 1986; Coleman, 1990; Ergincan, 2005; Kerler III \& Killough, 2009). De hecho, es una apuesta probabilística con información limitada, asimétrica y (a veces) contradictoria (Nooteboom, 2010). Se pone a prueba cuando, mientras que por un lado se carece de información de una situación específica, por otro se tiene la experiencia de éxitos y fracasos previos, y del contexto ampliado (Ruscio, 1996; Nooteboom, 2010). De ahí que el balance de ambas posiciones lleve al conflicto de decidir si se debe confiar o no (Nooteboom, 2010). En consecuencia, el acto de confiar conlleva cierta vulnerabilidad, toda vez que no se conocen los resolutivos de algo o alguien (Baier, 1986). Baier (1986) lo pone en términos de esperar que los otros no actúen con engaño aunque puedan hacerlo. Y Luhmann (1979) lo refiere como la espera de que lo mejor suceda, incluso aunque ello signifique apartarse de la razón. Así, la confianza y el riesgo se muestran como dos caras de la misma moneda, no se puede hablar de confiar sin que no se sobreentienda algún tipo de riesgo involucrado. Dicho de otro modo, en la medida en que uno aumenta, el otro disminuye.

De este modo, la confianza no es total ni categórica; existen niveles y graduaciones de ella (Nooteboom, 2010). Es contextual, condicional y puntual (Hardin, 2006; Hardin, 2010). Depende de quién se hable, de lo que se trate, de lo que involucre, de lo que se sepa, de las consideraciones internas y externas, de la reputación de las partes involucradas (Hardin, 2006), del orden de preferencias morales en cuestión (Uslaner, 2002), de ciertos supuestos emocionales (Lewis \& Weigert, 1985) y según a quien se dirija y deposite (Hardin, 2006; Hardin, 2010; Nooteboom, 2010). Por lo tanto, debe entenderse en un sentido coyuntural, tipológico y dimensional. Por eso se estudia desde cómo intervienen algunas de sus características, como el riesgo y la cooperación, en el desempeño efectivo de los negocios (Sengun \& Wasti, 2011); en cómo puede incidir en la innovación en un ambiente de competencia (Shama \& Elsawaby, 2014); en cómo impacta en el desempeño de las relaciones colaborativas (Paul \& McDaniel, 2004); y hasta en cómo interviene en el proceso de transferencia de conocimiento (Wang \& Zhong, 2014). 
En ese marco de la confianza, Nooteboom, (2010: 11) aborda una serie de cuestionamientos con fines clasificatorios: "¿En quién se puede confiar, en qué y con qué bases? ¿Quién es el depositario de la confianza? ¿En qué se puede confiar de las personas? ¿Cuál es el valor de la confianza? ¿Cuál es la base de la confianza?" En respuesta, refiere que se puede confiar en personas, objetos, procedimientos y organismos; tanto en hechos objetivos como en sustancias subjetivas interiorizadas y compartidas con otros. Adhiere que resulta complejo brindar nuestra confianza a un elemento que podríamos considerar indigno de ella; toda vez que -si se tiene en cuenta que en cada organismo o sistema, sus constituyentes y/o individuos que lo conforman, se encuentran en diversas posiciones y tienen diversos espacios de poder y de maniobra-, es de esperarse que cada uno de éstos actúe según sus objetivos, ya sean particulares o compartidos. Y éstos, a su vez, se deban a las presiones internas o externas; o bien a su carácter legítimo o ilícito.

Así, agrega que se confía en las competencias, compromisos e intenciones de las personas 0 entidades, que de igual modo asumimos como dignas de confianza. Empero que la decisión sobre quién debe ser el depositario de la confianza se sitúa en la dicotomía de si ésta simplemente se debe ofrecer "a cambio de nada", o es algo que alguien debe gradualmente "merecer o ganar" en relación con las expectativas y la experiencia vivida; $y$, con base en ello, actuar confiadamente o no. Es decir, se resuelve confiar como un hecho que deviene según nuestros juicios racionales, soportados con información y conocimiento; 0 , se debe simplemente actuar según nuestro "sentir" respecto de lo que el otro -objeto, persona o circunstancia en cuestión- indique. Esto es, si se entiende que la confianza es algo que se funda en lo moral y emocional (Lewis \& Weigert, 1985; Uslaner, 2002), o es una situación que es creciente en la medida de que sus fundamentos racionales también lo sean (Buck, 1985; Coleman, 1990; Williamson, 1993). De ahí que Cohen \& Dienhart (2012) expongan que la confianza es la base de las relaciones éticas organizacionales, dado que no siempre se encuentran balanceadas las expectativas sociales y los riesgos involucrados.

De esta forma, el mismo Nooteboom (2010) establece que la confianza se basa tanto en emociones; es decir, en condicionantes como el carácter, los sentimientos, las pasiones y los afectos (Lewis \& Weigert, 1985; Uslaner, 2002); como en fundamentos racionales, sustentados en información y análisis (Buck, 1985; Coleman, 1990; Williamson, 1993). Lewis \& Weigert (1985), por su parte, adhieren que la confianza está compuesta por aspectos cognitivos, emocionales y del comportamiento. Y, para el caso cognitivo, precisan que se vincula con un tipo de conocimiento que no necesariamente se funda en la razón, sino más bien en la experiencia práctica o en las creencias personales. En lo que respecta a las emociones, argumentan que se asocian fundamentalmente 
con el hecho de traicionar la confianza de algo o de alguien. Esto es, que se vincula estrechamente con la lealtad, y ésta solo se consigue cuando alguna de las partes renuncia a sus intereses fundados en la razón. Así, por último, la confianza ligada al comportamiento tiene que ver con el manejo de la incertidumbre, en el entendido de que quien confía actuará con la seguridad de que las cosas saldrán bien.

Más aún, Sztompka (1999) distingue que, en general, la confianza se constituye por siete elementos: regularidad, eficiencia, representatividad, justicia, responsabilidad, benevolencia y confiabilidad. La regularidad es lo que sucede con frecuencia y de la misma manera; bajo las mismas condiciones y criterios de puntualidad, en situaciones estables y periódicas, y con aspectos que lo precisan y lo caracterizan con uniformidad. La eficiencia, por su parte, es la capacidad para alcanzar los objetivos utilizando de forma óptima los recursos con los que se cuenta (Mihaiu, Opreaana \& Cristescu, 2010). La representatividad, entretanto, es el encuadre social que autoriza a los individuos actuar a nombre de otros (Abellán, 2013). Ello faculta el cimiento de organizaciones, instituciones y de sociedades enteras (Putman, 1993; Fukuyama, 1995; Ostrom \& Ahn, 2007). La justicia se integra de cuidado, eficiencia, lealtad, valores, principios, competencia y habilidades (Colquitt \& Rodell, 2011). Es ecuánime, coherente en su resolución, eficiente y eficaz de las disposiciones; y, además, honesta, recta, imparcial y objetiva respecto de los estatutos.

La responsabilidad, es cumplir con lo que se estipula. En relación con la confianza, es claro que si no se cumple, no se puede ser confiable. Así, la responsabilidad confiere la idea de seriedad, madurez y competencia respecto a algo y, proyecta compromiso, sensatez y buen juicio. La confianza, entonces, requiere ser responsable para la consumación de las obligaciones adquiridas (expectativas). Entretanto, la benevolencia es la capacidad de ser condescendiente, clemente, comprensivo y tolerante con los individuos. $Y$, en ciertas circunstancias, significa ser generoso e indulgente para ejercer la ley. Sin benevolencia, difícilmente la justicia, condición necesaria para la consolidación de la confianza, podrá ser honesta y equitativa (Colquitt \& Rodell, 2011). Por último, la confiabilidad tiene una actuación distintiva dentro de la noción de la confianza. Luhmann (2008) refiere que mientras que la confianza es algo por lo cual se puede decidir optar o no, la confiabilidad es una situación en la que no hay alternativas de elección, y sólo se puede desear que las cosas ocurran del modo en que se espera que lo hagan.

Paxton (1999), por consiguiente, menciona que la simple esperanza de que amanezca cada día, o la imposibilidad de negarse a pagar impuestos al gobierno -y que éstos sean bien administrados-, son ejemplos de confiabilidad. En cambio, Nooteboom 
(2010) la define como algo en lo que se tiene cierto control, toda vez que existe un contrato, reglamento o autoridad que da garantía de los resolutivos esperados. Es decir, desde esta perspectiva, la confiabilidad deviene del marco de instituciones que la procuran. No obstante, Hardin (2010) se aproxima más a Luhmann (2008) y la precisa como la realización de las expectativas. Esto es, si A espera que $B$ realice las acciones $X$ y $Z$, la consumación o no de tales acciones determinarán el grado de confiabilidad de B. De ahí que mientras la confianza es una elección, el nivel de confiabilidad reforzará o deteriorará lo oportuno de dicha decisión (Hardin, 2010).

De este modo, Nooteboom (2010) concluye que la confianza se compone de cuatro elementos: "El que confía, confía en aquél en quién deposita la confianza, en algún aspecto (competencia o intención) y según ciertas condiciones" (Nooteboom, 2010: 114). En otras palabras, el que decide confiar lo hace por y en algún objeto determinado, este puede ser un individuo o una institución; y lo hace porque le parece que sus intenciones son buenas y/o porque es competente de hacer lo que se espera que realice en una arena estipulada. Lo cual depende de otras variables contextuales que pueden ser de carácter emocional y racional (Nooteboom, 2010). Hardin (2010) añade que todos los tipos de confianza se insertan dentro de tres categorías genéricas: la confianza entre personas, instituciones y terceros (no instituciones); lo que puede ser simplificado como interpersonal e impersonal. De la primera delinea que se trata de un tipo de confianza de intereses encapsulados: 'yo confío en usted porque su interés encapsula el mío, lo que significa que usted tiene interés en satisfacer mi confianza. Lo que hace que mi confianza sea algo más que meras expectativas acerca de su conducta" (Hardin, 2010).

Por lo cual, el mismo Hardin (2010) deduce que dado que confiar es suponer que alguien tiene buenas intenciones para con nosotros, o bien un aspecto de nosotros -y que es competente para hacer, en lo que creemos que se desempeñará correctamente-, ergo, la confianza se compone tanto de "buenas intenciones" (motivaciones) como de competencias (habilidades y destrezas). En ese sentido, agrega que dichas "buenas intenciones" se derivan, a su vez, de dos circunstancias concretas: cuando existe el interés en cada una de las partes en mantener la relación, y cuando alguien tiene el potencial de ser confiable, ya sea por su nivel de responsabilidad moral o disposiciones propias de su carácter. Por consiguiente, infiere que la confianza encapsulada (interpersonal) es tripartita; es decir, "A confía en B para hacer X" (Hardin, 2010, p. 28); de naturaleza cognitiva: involucra simultáneamente conocimientos y creencias; y es gradual: incrementa o decrementa en la medida en que dicha confianza depositada es honrada o defraudada. De esta forma, puntualiza que una vez que se tiene un conocimiento relevante sobre alguien, según sea su deber moral y predisposición, 
tal conocimiento constituirá el grado de confianza con el cual nos dirigiremos hacia él o ella. Es decir, confiamos en alguien que visualizamos digno de confianza en un identificado espectro de tareas y acciones (Hardin, 2006).

\section{Tabla 1.4.1. La confianza interpersonal (encapsulada): Motivación y competencia}

\begin{tabular}{|l|lr|}
\hline \multirow{2}{*}{ A confía en B } & Para hacer & $\mathrm{X}$ \\
\cline { 2 - 3 } & Con respecto a & $\mathrm{X}$ \\
\hline
\end{tabular}

FUENTE: Hardin (2006)

En un segundo nivel, el aludido Hardin (2010) pone de relieve que situar a la confianza desde la perspectiva impersonal, significaría hacerlo más desde la óptica de la confiabilidad de las instituciones. Es decir, delibera que puesto que la confianza toma en cuenta tanto los motivos como el contexto en que la misma será puesta a prueba, suponer confiar en el gobierno y la sociedad, representa en términos simples confiar en cualquier persona o cosa respecto de cualquier aspecto. Por lo cual, argumenta que es inverosímil hablar de confiar en el gobierno, sino más bien, en que nuestras expectativas basadas en su desempeño sean satisfechas o no. Ejemplifica que si hubiera tal cosa como "confiar en el gobierno", significaría confiar en cada uno de los eslabones organizacionales que lo conforman; lo que implica conocer a cada una de las personas que a su vez las constituyen, entender sus diversas motivaciones, sus múltiples capacidades y mantener algún tipo de relación de intereses mutuos con ellas (Hardin, 2010).

Esto último es, de hecho, la principal razón por la cual el citado autor concluye que no existe tal cosa como la denominada confianza social o generalizada; rebatiendo así los trabajos de Putnam (1993) y Fukuyama (1995) que, por el contrario, asientan que los niveles de confianza compartida a una macro-escala incentivan la cooperación y el bienestar social. En otras palabras, dichos autores asumen que este tipo de confianza es un causal de orden y disposición funcional; definiéndose como la inclinación de los individuos a cooperar aunque no se conozcan entre sí (Stolle, 2002; Kouvo, 2011). Y asociándose con la actividad gubernamental (Stolle, 2002); con el devenir de los procesos históricos, sociales y políticos (Fukuyama, 1995; Putnam, 1993); y con el tipo y eficacia de las políticas sociales (Fukuyama, 2001). Además, fortalece y motiva la tolerancia entre los individuos; la regulación institucional disminuye la corrupción y mejora la eficiencia de la económica (Stolle, 2002). 
Más aún, Bjornskov (2006) encuentra que existe una relación inversamente proporcional entre la desigualdad económica, la diversidad étnica y la confianza generalizada; Paldam (2007) halla un nexo explícito entre la calidad de vida de una región y los niveles de confianza generalizada; Ozcan \& Bjornskov (2011), identifican un vínculo inmediato entre el estado de desarrollo humano de una localidad y la confianza generalizada; Olsen (2008) delinea una asociación entre el nivel de confianza social y los incentivos para invertir; Bac (2009), deduce una correspondencia positiva entre la confianza generalizada y las políticas de distribución y bienestar; Bjornskov (2009) liga el nivel de escolaridad de una población con el de confianza social; Lindstrom (2009) halla que las condiciones laborales y el desempleo se asocian con el grado de confianza generalizada; y Fairbrother \& Martin (2012), por último, demuestran que la desigualdad económica disminuye la capacidad de confiar en otros.

De donde, es claro que la forma agregada de la confianza tiene un rol en los resolutivos sociales. De hecho, en ambas unidades de análisis, interpersonal e impersonal, la confianza actúa positivamente en solventar los problemas de coordinación. Putnam (1993) sugiere que la interacción positiva a un micro-nivel en algún punto puede generalizarse, lo que se traduciría en una especie de puente entre ambos niveles. No obstante, debe tenerse en cuenta que la confianza, después de su inter e impersonalidad genérica, contiene una dimensión gradual, contextual, condicional y coyuntural; y adquiere una forma racional, moral, emocional, cultural y cognitiva; que derivan, a su vez, en la creación de redes, instituciones y normas de reciprocidad: el logro de la acción colectiva (Ostrom \& Ahn, 2007). Deben considerarse también sus características definitorias, como lo son sus componentes de proximidad psicosocial, su rol en las creencias compartidas, la singularidad de los incentivos que la motivan, la contextura de las amenazas y riesgos que la enmarcan y delimitan, su sentido de justica, los condicionales que promueven actuar desconfiadamente como resultado de experiencia previas, su proyección hacia la lealtad, su interacción en la lógica de éxito y fracaso; y su contrasentido en la información que se tiene y que envuelve la toma de decisiones. 
Tabla 1.4.2. La confianza:

Dimensiones, sus aspectos operativos y lo que involucra

\begin{tabular}{|c|c|c|}
\hline Dimensiones & Operatividad & Se caracteriza o involucra \\
\hline $\begin{array}{l}\text {-Gradual, no categórica } \\
\text {-Específica } \\
\text {-Contextual } \\
\text {-Condicional } \\
\text {-Coyuntural } \\
\text {-Racional } \\
\text {-Emocional } \\
\text {-Moral } \\
\text {-Cultural } \\
\text {-Cognitiva }\end{array}$ & $\begin{array}{l}\text {-Logro de la acción } \\
\text { colectiva } \\
\text {-Redes } \\
\text {-Instituciones } \\
\text {-Normas de reciprocidad }\end{array}$ & $\begin{array}{l}\text {-Proximidad psicosocial } \\
\text {-Creencias compartidas } \\
\text {-Una apuesta probabilística } \\
\text {-Un conocimiento evaluado } \\
\text {-Una estimación futura } \\
\text {-Riesgo } \\
\text {-Responsabilidad } \\
\text {-Estabilidad } \\
\text {-Experiencias previas } \\
\text {-Incentivos } \\
\text {-Expectativas } \\
\text {-Intereses compartidos } \\
\text {-Vulnerabilidad } \\
\text {-Algún tipo de relación } \\
\text {-Ser competente } \\
\text {-Éxito o fracaso } \\
\text {-Lealtad } \\
\text {-Cumplimiento de normas y } \\
\text { leyes } \\
\text {-Incertidumbre } \\
\text {-Disposición de carácter } \\
\text {-Una paradoja de información } \\
\text {-Regularidad } \\
\text {-Representatividad } \\
\text {-Eficiencia } \\
\text {-Justicia } \\
\text {-Benevolencia } \\
\text {-Confiabilidad }\end{array}$ \\
\hline
\end{tabular}

FUENTE: Elaboración propia con datos de Lewis \& Weigert (1985), Baier (1986), Coleman (1990), Putnam (1993), Misztal (1996), Hardin (2006, 2010), Ostrom \& Ahn (2007), Luhmann (2008), Nooteboom (2010) y Adler -Lomnitz (2012). 


\section{Aproximación teórica al efecto producto del encadena- miento inseguridad personal - desconfianza generalizada - diferencias delictivas comunitarias}

Desde la perspectiva de ATD, las teorías que lo conforman van de la situación causal al crimen consumado, como si en la mente de los individuos no acontecieran una serie de conflictos, dificultades o tensiones, que si bien se dejan entrever en las referidas aproximaciones, no profundizan en ello. Es decir, no necesariamente serían las condiciones del entorno lo que derivaría en el acto delictivo como tal, sino más bien cómo los individuos lo asimilan y responden ante él.

Figura 2.1. Proceso del crimen por ATD: entorno, cognición y reacción

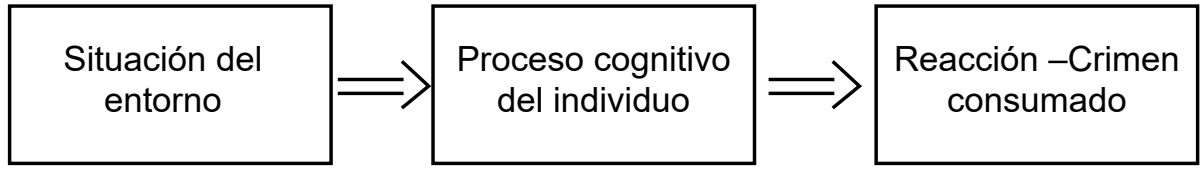

Fuente: elaboración propia

De ahí que cuando se toma cuenta: (1) que las causas de la inseguridad personal provienen de las dificultades para sobrevivir y el poder hacer elecciones; (2) que ATD arguye que las carencias materiales, la segregación, la marginación, los débiles lazos normativos y comunitarios, son los determinantes del crimen (Merton, 1968; Agnew, 1992; Bourffard \& Muftic, 2006); (3) y que con la intención de dar certidumbre a sus acciones, el hombre crea artificios colectivos denominados instituciones, mismas que no estaría dispuesto a seguir si en algún punto éstos no se percibieran seguros (Campbell, 2009). Resultaría, por tanto, que los factores que condicionan la percepción de sentirse inseguro podrían ser los mismos que motivan al delito; y que los constituyentes que originan la creación del Estado, el gobierno y la familia (Hobbes 2009; Eroglu, 2012), serían, de hecho, los mismos que incitarían a la acción criminal.

De modo tal que si se piensa en la seguridad como la primera búsqueda del ser humano (Williams, 2013), y como un determinante en la creación de instituciones (Laso, 2007; Hobbes 2009; Eroglu, 2012), debe concebírsele también como un estado de desconfianza que conduciría hacia un tercer estadio en el entendimiento del delito: el crimen por inseguridad personal. Refiriéndose, en un primer momento, a la consideración de los factores materiales, normativos e institucionales que rodean al individuo e inciden en su decisión; y a las 
tensiones internas que éstas le generan, en una segunda aproximación (Merton, 1968; Agnew, 1992). El tercero, del que se habla, insinuaría precisamente el análisis de la naturaleza de dicho componente cognitivo: un tipo de desconfianza vinculado con la percepción de inseguridad.

Más aún, hay razones empíricas que obligan a pensar en una estrecha proximidad entre la (des)confianza y el crimen. A decir de ello, considérese, por ejemplo, que el capital social, definido como las pautas relacionadas con la colaboración y contribución efectiva entre los individuos de una comunidad (Ostrom, 1990; Fukuyama, 1995), se encuentra íntimamente vinculado con la confianza (Ostrom \& Anh, 2007). No es casual que vasta literatura científica asocie directamente a uno y otro (Rosenfeld, Messner \& Baumer, 2001; Lederman et al. 2002; Deller \& Deller, 2010; Vieno et al. 2010; Hall et al. 2014). De ahí que si la confianza es el elemento indispensable en la conformación del así llamado capital social (Ostrom \& Anh, 2007), se infiera que también tenga un rol preponderante con la intención criminal.

De igual forma, obsérvese que un alto grado de desconfianza social se asocia con la pobreza, la desigualdad económica, el desempleo, la marginación social, la baja escolaridad y la heterogeneidad étnica y racial (Bjornskov, 2006; Paldam, 2007; Bjornskov, 2009; Lindstrom, 2009; Ozcan \& Bjornskov, 2011; Wikes, 2011; Fairbrother \& Martin, 2012; Hooghe, Merien \& Vroome, 2012); con la mala calidad de los servicios públicos y deficientes políticas sociales y distributivas (Fukuyama, 2001; Larsen, 2007; Bac, 2009); y, con las creencias y emociones de los individuos (Dunning, Fetchenhauer \& Schlosser, 2012). Mientras que la propensión criminal por ATD, también se interconecta con los mismos catalizadores (Merton, 1968; Wilson \& Kelling, 1982; Gottfredson \& Hirschi, 1990; Agnew \& White, 1992; Hagan, 1992; Hirschi, 1995; Mosher, Miethe \& Phillips, 2002; Hollist, Hughes \& Schaible, 2009; Manasse \& Ganem, 2009; Jennings, Piquero, Gover \& Pérez, 2009; Ibabe \& Jaurewuizar, 2010; Schroeder, Hill, Haynes \& Bradley, 2011; Sigfusdottir, Kristjansson \& Agnew, 2012).

Lo anterior, lleva a entrever una conexión entre el crimen por ATD, la confianza y la confianza genera lizada. En este sentido, dado que esta última se define como el grado con el cual se percibe contar con los demás aunque no se les conozca (Stolle, 2002; Kouvo, 2011), ello implica suponer que es dable tener cierto grado de predictibilidad sobre el comportamiento de los que no son cercanos. Algo que Hardin (2006) objeta bajo el argumento de que dado que todos los tipos de confianza son puntuales, graduales, contextuales y condicionales -y que, además, son de naturaleza tripartita; es decir, se confía en alguien (o algo), en una determinada arena, o en un reducido campo de acción-, confiar sin que se tenga previo conocimiento de las cualidades o competencias de un objeto o persona en cuestión, significaría el despropósito de confiar en cualquier entidad, en relación a cualquier cosa que se ocurra. 
Tabla 2.1 Causas de la (des)confianza generalizada y la propensión delictiva por ATD

\begin{tabular}{|c|c|}
\hline $\begin{array}{c}\text { Causales de la (des)confianza } \\
\text { generalizada }\end{array}$ & $\begin{array}{c}\text { Causales de la propensión al delito } \\
\text { por ATD }\end{array}$ \\
\hline $\begin{array}{l}\text { Pobreza, desigualdad, } \\
\text { desempleo, marginación, baja } \\
\text { escolaridad, heterogeneidad } \\
\text { étnica y racial. }\end{array}$ & - $\begin{array}{l}\text { Pobreza, desigualdad, desempleo, } \\
\text { marginación, baja escolaridad, } \\
\text { heterogeneidad étnica y racial. }\end{array}$ \\
- $\begin{array}{l}\text { Servicios públicos ineficientes } \\
\text { y deficientes políticas sociales } \\
\text { y distributivas. }\end{array}$ & - $\begin{array}{l}\text { Abandono de la comunidad. } \\
\text { Creencias y aspectos } \\
\text { emocionales de los individuos. }\end{array}$ \\
\end{tabular}

FUENTE: Elaboración propia con datos de Fukuyama (2001), Mosher, Miethe \& Phillips (2002), Fajnzylber, Lederman \& Loayza (2002), Bjornskov (2006), Paldam (2007), Larsen (2007), Ramírez (2008), Bjornskov (2009), Lindstrom (2009), Bac (2009), Deller \& Deller (2010), Kouvo (2011), Dunning, Fetchenhauer \& Schlosser (2012) y Hooghe, Merien \& Vroome (2012).

Cabe señalar que también debe considerarse que la (des)confianza generalizada puede estudiarse desde otras avenidas. Por ejemplo, mientras hay trabajos que la vinculan haciendo énfasis en los factores materiales (Fukuyama, 2001; Larsen, 2007; Bac, 2009), otros puntualizan que la desconfianza social resulta de la violencia y la alta incidencia delictiva (Walklate, 1998; Lederman, Loayza \& Menéndez, 2002; Kruger, Hulchison, Monroe, Reischl \& Morrel-Samuels, 2007; Gainey, Alper \& Chappell, 2011; Clark \& Eisenstein, 2012); o que el crimen inhibe la confianza comunitaria y por ende la cooperación social (Sampson, 2001; De Coster, Heimer \& Wittrock, 2006; Deller \& Deller, 2010). En tanto otra corriente de pensamiento se centra en el análisis de los mecanismos que promueven la confianza entre los ciudadanos, los cuerpos de seguridad y las instituciones de justicia (Devos, Spin \& Schwartz, 2002; Goldsmith, 2005; Weitzer \& Tuch, 2005; Allen, Edmons, Patterson \& Smith, 2006; Kaarianinen, 2008); y los que señalan, desde luego, que la desconfianza comunitaria resulta de la falta de oportunidades para el desarrollo (Bjornskov, 2006; Paldam, 2007; Bjornskov, 2009; Lindstrom, 2009; Ozcan \& Bjornskov, 2011; Wikes, 2011; Fairbrother \& Martin, 2012; Hooghe, Merien \& Vroome, 2012).

Aunado a lo anterior, inclusive debe tomarse en cuenta que dado que: (1) la institución denominada Estado tiene como objeto garantizar el bienestar, la protección y el resguardo de los individuos, resulta ser una estrategia de sobrevivencia colectiva (Estrada, 2013); 
(2) el acto de confiar en las personas de una comunidad implica hacer un juicio ampliado del marco de leyes, reglamentos, organizaciones, historia y cultura que se supone inciden en su comportamiento (Putnam, 1993; Fukuyama, 1995; Stolle, 2002); es claro que en ambos casos la confianza generalizada significaría tener expectativas positivas de los individuos, tanto por las creencias que se comparten (instituciones informales), como por la confiabilidad de las instituciones políticas que se supone regulan su actuación.

En síntesis, se puede afirmar que:

$\checkmark \quad$ Las instituciones se conforman por valores; y éstos a su vez, proveen seguridad física, capacidades y una noción de lo apropiado (justicia) a los individuos para su actuación social confiada.

$\checkmark$ ATD dicta que la desigualdad, la exclusión, la segregación y la frustración social, son los causales del crimen.

$\checkmark \quad$ La inseguridad se debe tanto a las carencias materiales y débiles capacidades sociales, como a las amenazas del entorno y de otros.

Figura 2. 2. Triangulación entre los causales del delito por disfunción social, los efectos del mal funcionamiento institucional y las causas de la inseguridad

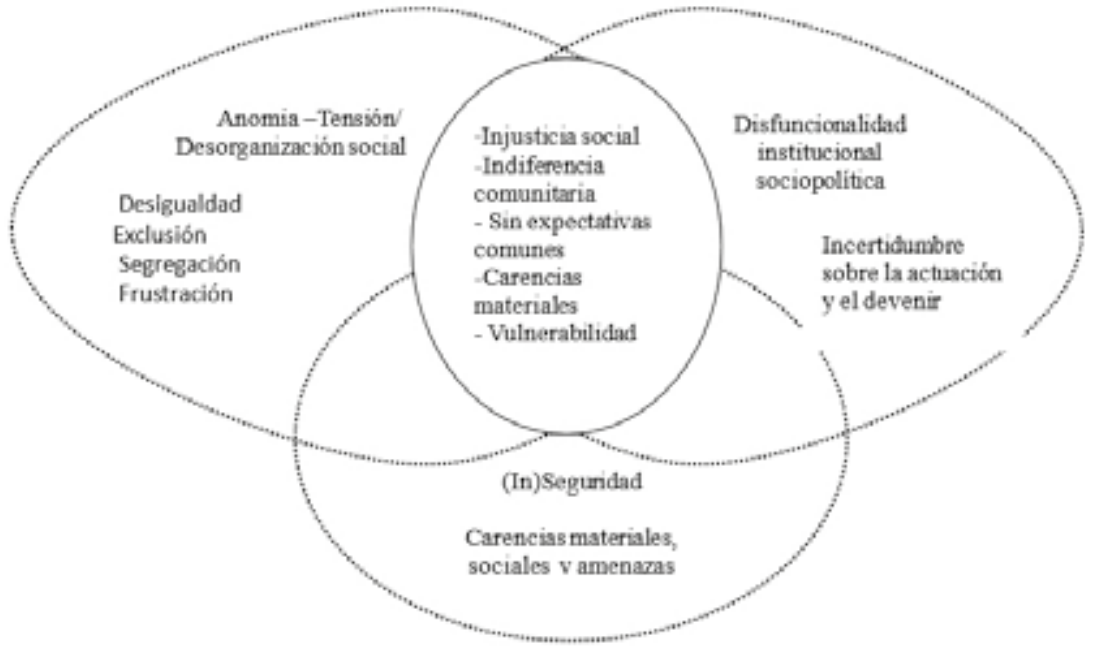

FUENTE: Elaboración propia con datos de Merton (1968), Agnew (1992), Deller \& Deller (2010), Rock (2012) y Williams (2013) 
A partir de la triangulación de cada uno de estos aspectos, sus factores comunes hablan sobre las siguientes necesidades de los individuos en dos momentos: (1) material y resguardo de las amenazas del entorno y de otros; (2) sentido de lo apropiado o de justicia, cercanía comunitaria, percibir que se tienen expectativas de beneficio común. No obstante, si se descuenta el aspecto material y las amenazas, dado que no son de naturaleza cognitiva, y se contrastan los factores restantes con los siete elementos constituyentes de la confianza de Sztompka (1999), es notorio que los determinantes obtenidos pueden ser expresados en términos de desconfianza, a saber:

Tabla 2. 2. Factores que convergen en la propensión criminal, la inseguridad y la disfunción institucional; y su equivalencia con la desconfianza

\begin{tabular}{|c|l|l|l|}
\hline $\begin{array}{l}\text { Elementos en } \\
\text { común de la } \\
\text { disfuncionalidad } \\
\text { institucional, la } \\
\text { anomia tensión y } \\
\text { desorganización } \\
\text { social, y la } \\
\text { inseguridad. }\end{array}$ & $\begin{array}{l}\text { La percepción de } \\
\text { Injusticia social }\end{array}$ & $\begin{array}{l}\text { La indiferencia } \\
\text { comunitaria } \\
\text { respecto a las } \\
\text { dificultades } \\
\text { personales }\end{array}$ & $\begin{array}{l}\text { La apreciación de } \\
\text { que no se tienen } \\
\text { expectativas de } \\
\text { beneficio común }\end{array}$ \\
\hline $\begin{array}{l}\text { Elementos de la } \\
\text { (des) confianza }\end{array}$ & Injusticia & $\begin{array}{l}\text { No } \\
\text { presentatividad } \\
\text { Malevolencia }\end{array}$ & $\begin{array}{l}\text { Irregularidad } \\
\text { Ineficiencia } \\
\text { No confiabilidad } \\
\text { Irresponsabilidad }\end{array}$ \\
\hline
\end{tabular}

FUENTE: Elaboración propia con datos de Sztompka (1999)

De esta forma, resulta asequible plantear un posible mecanismo funcional del crimen por desconfianza o inseguridad personal. Esto es, en un estado inicial, acontecería que los individuos se sienten desconfiados en relación con las dificultades inherentes para asegurar su sobrevivencia; la cual tiene una dimensión física y una social. La primera compete a las necesidades materiales, tales como alimento y vestido, así como a la protección de las amenazas del entorno y de los posibles daños que otros les puedan infligir. Mientras que la segunda, se abocaría a las necesidades de afecto y reconocimiento (Merton, 1968; Agnew, 1992), y de contar con la capacidad para poder hacer elecciones (Williams, 2013). 
Figura 2.3. Mecanismo del crimen por desconfianza

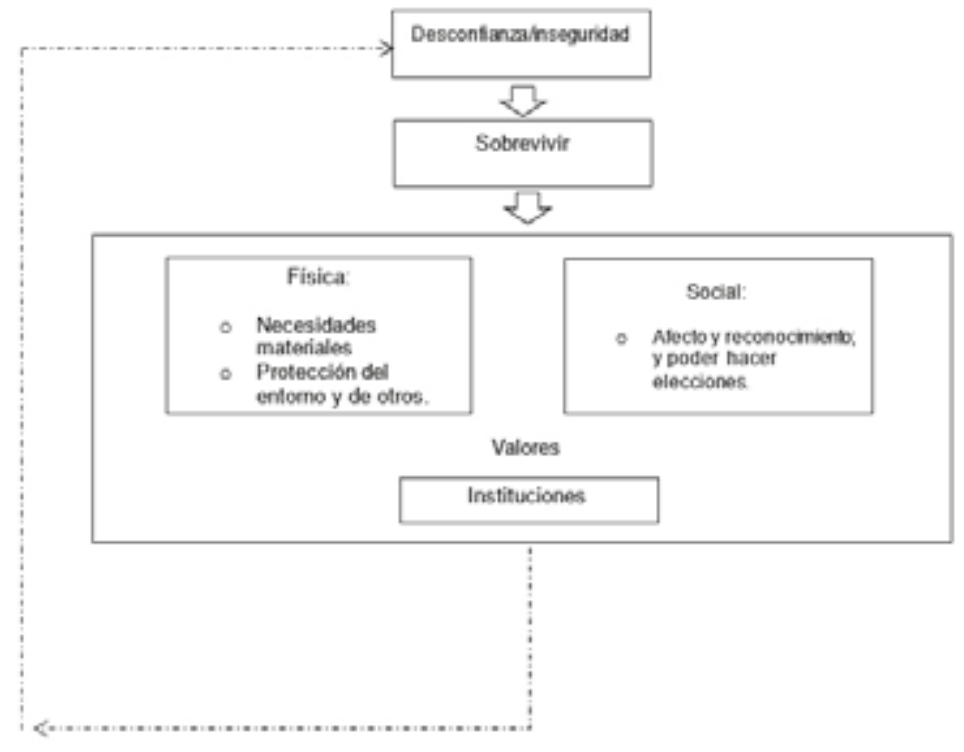

FUENTE: Elaboración propia

Sin embargo, la literatura identifica tres tipos de inseguridades por disfuncionalidad institucional vinculadas con la noción de estar seguro, y que conforman el estado total de la confianza generalizada de una comunidad:

1. Desconfianza por carencia material (Fukuyama, 2001; Larsen, 2007; Bac, 2009 Bjgrnskov, 2006; Paldam, 2007; Lindstrom, 2009; Ozcan \& Bjqrnskov, 2011; Fairbrother \& Martin, 2012).

2. Desconfianza por amenazas del entorno y de otros (Walklate, 1998; Lederman, Loayza \& Menéndez, 2002; Kruger, Hulchison, Monroe, Reischl \& Morrel-Samuels, 2007; Gainey, Alper \& Chappell, 2011; Clark \& Eisenstein, 2012).

3. Desconfianza por falta de afecto, reconocimiento y capacidades (Betts, Rotenberg \& Trueman, 2009; Dunning, Fetchenhauer \& Schlosser, 2012; Bjornskov, 2009; Lindstrom, 2009; Wikes, 2011; Fairbrother \& Martin, 2012; Hooghe, Merien y Vroome, 2012).

De estas, las que producirían el crimen por ATD, son 1 y 3 , aunque preponderantemente esta última alude al constructo teórico al que se hace referencia; y es por ello, además, que si bien sus determinantes son la carencia material, tiene más peso la percepción de injustica social, de indiferencia comunitaria y de percibir que "los otros" no tienen expectativas de beneficio común. 
Resulta así que la inseguridad personal que conllevaría al crimen por ATD y sus diferencias comunitarias, se basaría en la idea de que un grupo de individuos comparten una desconfianza relacionada con las dificultades materiales y sociales que los circundan. Esto determina que su medición considere, simultáneamente, tanto el grado de confiabilidad de las instituciones formales, como el de las informales, en las que dichos sujetos hacen su apuesta por su seguridad.

Esto debe entenderse, por un lado, en el contexto de la seguridad humana; toda vez que la democracia liberal es el paradigma dominante de los valores que enmarcan la institucionalidad formal. Es decir, su significado y alcance se logran cuando se consigue la eliminación de todas las amenazas que vulneran la dignidad del ser humano desde la perspectiva de la democracia liberal. A una arena social percibida como injusta, indiferente a las dificultades personales y sin esperanzas de mejora común, se la debe situar en un entorno con falta de oportunidades y desigualdades económicas y sociales, tales como el desempleo, la pobreza, la desnutrición, la carencia de servicios básicos y educativos, la marginación, la inseguridad social, la discriminación, la desunión familiar y étnica, y sin las garantías ni las libertades para el ejercicio de los derechos humanos (PNUD, 2014).

No obstante, también se la debe pensar en el sentido de una seguridad de enfoque construccionista. Esto es, dado que la seguridad es la ausencia de cualquier tipo de amenaza que comprometa la sobrevivencia desde los encuadres propios de la comunidad, una sociedad concebida como injusta, indiferente y sin expectaciones comunes, tendrá parámetros endógenos diferenciados de lo que es una amenaza o riesgo, injusto y desesperanzador. $Y$ si bien la diferencia entre ambas posiciones es sutil, es importante mencionarla, toda vez que puede caerse en el error de utilizar mediciones como el nivel de ingreso o la simple confianza en el gobierno; o bien, cuantificar ambas perspectivas en forma separada y con instrumentos no construidos desde la psicología social, los cuales dominan la investigación de la confianza generalizada (Hardin, 2006).

En síntesis, el delito por desconfianza sugiere que un individuo percibe que no cuenta con el apoyo de los otros para sobrevivir y realizarse socialmente. Así, la disfuncionalidad de las instituciones formales e informales promueve la noción individual, después compartida, de que el sistema es injusto, indiferente a la dificultad personal y carente de esperanzas. Toda la vasta evidencia que acompaña a ATD ha omitido el simple hecho de centrar su atención en el proceso individual cognitivo en vez de los factores físicos e institucionales del entorno. Y más aún, cuando por dicho efecto pueden hallarse diferencias delictivas entre las comunidades, es dable hablar de un tipo de inseguridad o desconfianza micro que construye crecientes formas macro del crimen. 


\section{Conclusiones}

El efecto producto del encadenamiento inseguridad personal - desconfianza generalizada - diferencias delictivas comunitarias, supone lo siguiente:

1. La propensión delictiva por ATD se suscita por un problema de inseguridad personal que deriva de las dificultades del entorno.

2. La cognición individual que sobreviene de dicha inseguridad es una de desconfianza, que se vincula con el problema sobrevivir y el poder hacer elecciones.

3. Tal desconfianza que es micro, al generalizarse en forma creciente, hace que sobrevengan las diferencias delictivas comunitarias. Así, el punto uno se sostiene sobre los factores que conllevan a la inseguridad personal y al delito por ATD: pobreza, desigualdad, desempleo, marginación, rechazo, discriminación, desunión familiar y étnica. El punto dos, por otro lado, hace hincapié en que se trata de una cognición, porque involucra tanto la razón como las creencias; y es desconfianza, toda vez que refiere a una estimación futura, negativa y riesgosa de un medio que se percibe como injusto, malévolo e ineficiente respecto de "los otros" y las instituciones que inciden en su actuación. La puntualización tres, en tanto, supone que dicha desconfianza individual en algún punto se generaliza. Es decir, si las instituciones son un conocimiento compartido que dicta cómo las cosas se hacen, ¿por qué la desconfianza individual no podría ser compartida del mismo modo en que éstas lo hacen?; o bien, ¿por qué una cognición individual respecto de un ambiente depresivo no sería conllevada por individuos con formas de interacción, comunicación y grados inteligibles similares? De hecho, tanto en uno como en otro sentido, no compartir un juicio sobre un contexto que involucraría al "sujeto y los otros", sería más bien menos coherente si se toma en cuenta que la citada cognición individual implica -si bien hechos en apariencia objetivos- también creencias y valores que "los otros" (la estructura) ha (n) insertado como parámetros analíticos en dicho individuo. De modo que, al ser compartida, este tipo de desconfianza generaría efectos agregados diferenciables, tal y como sucede con otros fenómenos que acontecen entre las sociedades con grados disimiles de igualdad política, económica y social.

Así, el encuadre teórico que se ha formulado se convierte en una bisagra entre la importancia de la calidad de las instituciones políticas y sociales, las causas de la propensión al delito por ATD y el abordaje mismo de la seguridad desde una perspectiva amplia: la seguridad humana, construccionista y los estudios de paz, que se suscriben a la noción de que los factores ambientales e institucionales son los causales de la inseguridad. 
Ahora bien, centrar la atención en lo cognitivo que deviene de los condicionales externos que no se procuran, conlleva al entendimiento de un "impulso con un potencial criminal" subyacente en la naturaleza humana: la lucha por la sobrevivencia y la búsqueda de las capacidades sociales.

Considerando que la gran mayoría de los enfoques teóricos del crimen se han abocado más en cómo hacer que las instituciones -Estado, gobierno, nación, comunidad, etcéterasean afectivas, se ha aplazado dilucidar propiamente por qué dichos mecanismos tienen su origen en la mente humana.

Finalmente, la idea de que la inseguridad de unos sea producto de la inseguridad de otros, abona a una discusión en el sentido más básico de las estrategias de prevención y contención del crimen. Una (des)confianza de este tipo, si bien rodeada por una vasta disputa sobre la mejor manera de su cálculo y control, puede ser medible y monitoreada con relativa eficiencia; lo que abriría nuevas posibilidades a los diseñadores e instrumentadores de políticas públicas en materia de seguridad ciudadana. 


\section{REFERENCIAS}

Abellán, P. (2013). Representación política y democracia: Aportaciones desde la teoría de la representación en los últimos diez años. Revista de Ciencia Política, 33, 133 - 147.

Adler-Lomnitz, L. (2012). Redes sociales, cultura y poder: ensayos de antropología latinoamericana. México: Flacso.

Agnew, R. (1992). Foundations for a General Strains Theory. Criminology, 30(1), 47-87.

Agnew, R. \& White, H. (1992). An Empirical Test of General Strain Theory. Criminology, 30 (4), 475-99.

Allen, J., Edmonds, S., Patterson, A. \& Smith, D. (2006). Policing and the Criminal Justice System, Public Confidence and Perceptions: British Crime Survey. Recuperado de www.homeoffice. gov.uk <última fecha de consulta, 3 de noviembre de 2016>

Bac, M. (2009). Generalized trust and wealth. International Review of economics, 29, 46-56.

Baier, A. (1986). Trust and Anti-Trust. Ethics, 96(2), 231-260.

Beltrame, F. (2011). Seguridad ciudadana y nuevas estrategias de control del delito en Argentina. Revista Latinoamericana de Seguridad Ciudadana, 9, 102-112.

Betts, L.R., Rotenberg, K.J. \& Trueman, M. (2009). The early Childhood Generalized Trust Belief Scale. Early Childhood Research Quarterly, 24, 175-185.

Bilgin, P. (2013). Critical Theory. En P. D. Williams (Ed.), Security Studies: An introduction (pp. 93-107). New York, EUA: Routledge.

Booth, K. (1991). Security and emancipation. Review of International Studies, 17(4): 313 - 26.

Bouffard, J. \& Muftic, L. (2006). Program Completion and Recidivism Outcome among Adult Offenders Ordered to Complete a Community Service Sentence. Journal of Offender Rehabilitation, 43(2), $1-33$.

Bjornskov, C. (2006). Determinants of generalized trust: A cross-country comparison. Public Choice, 130, 1-21.

Bjornskov, C. (2009). Social trust and the growth of schooling. Economics of Education Review, $28,249-257$

Buck, A. (1985). An empirical note on the Foundations of Rational Expectations. Journal of post Keynesian Economics, 7(3).

Clark, A.K. \& Eisenstein, M.A. (2012). Interpersonal trust: An age-period-cohort analysis revisited. Social Science Research, 42, 361-375.

Campbell, J. L. (2009). Surgimiento y transformación del análisis institucional; en E. Ibarra (Coordinador), Estudios Institucionales (pp. 3 -28). México: Editorial Gedisa.

Cohen, M. \& Dienhart, J. (2012). Moral and Amoral Conceptions of Trust, with an Application in Organizational Ethics. J Bus Ethics, 112, 1 - 13.

Coleman, J. S. (1990). Foundations of Social Theory. Massachusetts, EUA: Harvard University Press. 
Collins, A. (Ed.). (2013). Introduction: What is Security Studies? Contemporary Security Studies (pp. 1 - 13). Reino Unido: Oxford University Press.

Colquitt, J. \& Rodell, J. (2011). Justice, Trust, and Trustworthiness: A longitudinal Analysis Integrating Three Theoretical Perspectives. Academy of Management Journal, 54(6), 118 -1206 .

De Coster, S., Heimer, K. \& Wittrock, S.M. (2006). Neighborhood disadvantage, social capital, street context, and young violence. The sociological Quarterly, 47(4), 723 - 753.

Deller, S. C. \& Deller, M. A. (2010). Rural Crime and Social Capital. Growth and Change, 41(2), 221-275.

Devos, T., Spini, D. \& Schwartz, S. (2002). Conflicts among Human Values and Trust in Institutions. British Journal of Social Psychology, 41(4), 481-484.

Dunning, D. Fetchenhauer, D. \& Schlosser, T.M. (2012). Trust as social and emotional act: Noneconomic considerations in trust behavior. Journal of Economics Psychology 33(3), 686-694.

Ergincan, Y. (2005). Protecting Investors and lessening Moral Hazard in Capital Markets by Using Mobile Communications and Internet Technologies and The Role of Central Registry Agency of Turkey. Journal of Economic \& Social Research, 7, 31 -50.

Eroglu, S. E. (2012). Values: Great challenge for construction of social structure with social institutions. International Journal of Human Sciences, 9(2), $82-90$. Recuperado de http://www.j-humansciences.com/ojs/index.php/IJHS/article/view/2169<última fecha de consulta, 3 de noviembre de 2016>

Estrada, J.L. (2013). Democracia, Estado y Seguridad Ciudadana. Alegatos, 84.

Fairbrother, M. \& Martin, I. (2012). Does inequality erode social trust? Results from multinivel models of US states and countries. Social Science Research, 42(2), 347-60.

Fajnzylber, P., Lederman, D. \& Loayza, N. (2002). Inequality and violent crime. Journal of Law and Economics, 45, 1-40.

Fligstein, N. (2001). Social Skills and Theory of Fields. Sociological Theory, 19, 105-25.

Fukuyama, F. (1995). Trust: The social virtues and the creation of prosperity. Nueva York, EUA: The Free Press.

Fukuyama, F. (2001). Social Capital, Civil Society and Development. Third World Quarterly, 22, $7-20$.

Gainey, R., Alper, M. \& Chappell, A. (2011). Fear of crime Revisited: Examining the Direct and Indirect Effects of Disorder, Risk Perception, and Social Capital. American Journal of Criminal Justice, 36(2), 120-137.

Gambeta, D (Ed.). (2008). Can we Trust Trust?; en, Gambeta, D. (Ed.) Trust: Making and Breaking Cooperative Relations (pp. 213 -239). EUA: Basil Black Wells, Oxford University Press.

Goldsmith, A. (2005). Police Reform and the problem of trust. Theoretical Criminology, 9(4), 443470.

Gottfredson, M. \& Hirshi, T. (1990). A General Theory of Crime. California, EUA: Stanford University Press.

Hagan, J. (1992). The poverty of a classless criminology. Criminology, 30, 1-18. 
Hall, B.J., Bolton, P.A., Annan, J., Kaysen, D., Robinette, K., Cetinoglu, T., Wachter, K. \& Bass, J.K. (2014). The Effect of Cognitive Therapy on Structural Social Capital: Results from a Randomized Controlled Trial Among Sexual Violence Survivors In the Democratic Republic of the Congo. American Journal of Public Health, 104(9).

Harbeck, K.M. (2009). Social Disorganization Theory. Recuperado de http://www.academicpub. com/map/items/29538.html

Hardin, R. (2006). Trust. Massachusetts, EUA: Polity Press.

Hardin, R. (2010). Confianza y confiabilidad. México: Fondo de Cultura Económica.

Hirschi, T. (1995). La familia. San Francisco, EUA: Estudios Contemporáneos.

Hobbes, T. (2009). Leviatán o la materia, forma y poder de un Estado eclesiástico y civil. España: Alianza Editorial.

Hooghe, M., Merien, S. \& Vroome, T. (2012). The cognitive basis of trust. The relation between education, cognitive ability, and generalized and political trust. Intelligence, 40(6), 604-613.

Ibabe, I. \& Jaureguizar, J. (2010). Child to parent violence: Profile of abusive adolescents and their families. Journal of Criminal Justice, 38(4), 616 - 624.

Jennings, W., Piquero, N., Gover, A. \& Pérez, D. (2009). Gender and general strain theory: A replication and exploration of Broidy and Agnew's gender/strain hypothesis among a sample of southwestern Mexican American adolescents. Journal of Criminal Justice, 37(4), 404-417.

Kaarianinen, J. (2008). "Why Do the Finns Trust the Police?”. Journal of Scandinavian Studies in Criminology and Crime Prevention, 9(2), 141-159.

Kahan, D. (2003). The logic of reciprocity: trust, collective action, and law. Michigan Law Review, 102,71 .

Kelly, M. (2000). Inequality and crime. Review of Economics and Statistics, 82(4), 530-59.

Kerler III, W. \& Killough, L. (2009). The Effects of Satisfaction with Client's Management During A prior Audit Engagement, Trust and Moral Reasoning on Auditors' Perceived Risk of Management Fraud. Journal of Business Ethics, 85(2), 109 -136.

Kouvo, A. (2011). The sources of generalized trust and institutional confidence in Europe. Research on Finnish Society, 4, 29-40.

Kramer, R. (2012). Collective Trust within Organizations: Conceptual Foundations and Empirical Insights. Corporate Reputation Review, 13, 82 -97.

Kruger, D.J, Hulchison, P., Monroe, M.G., Reischl, T. \& Morrel-Samuels, S. (2007). Assault Injury Rates, Social Capital, and Fear of Neighborhood Crime. Journal of Community Psychology, 35(4), 483-498.

Larsen, C. A. (2007). How welfare regimens generate and erode social capital: The impact of underclass phenomena. Comparative Politics, 40, 83-1001.

Laso, E. (2007). Las instituciones desde la perspectiva psicológica: el punto de vista evolutivo. Recuperado de www.psicologiaenpositivo.com <última fecha de consulta, 3 de noviembre de 2016>

Lederman, D., Loayza, N. \& Menéndez, A.M. (2002). Violent Crime: Does Social Capital Matter? Chicago, EUA: World Bank, University of Chicago. 
Lewis, D. \& Weigert, A. (1985). Trust as a Social Reality. Social Forces, 63(4).

Lindstrom, M. (2009). Psychosocial work conditions, unemployment, and generalized trust in other people: A population- based study of psychosocial health determinants. The social Science Journal, 46(3), 584-593.

Lubell, M. (2007). Familiarity Breeds Trust: Collective Action in a Policy Domain. The Journal of Politics, 69(1), $237-250$.

Luhmann, N. (1979). Trust and Power. Chichester, Reino Unido: John Wiley \& Sons

Luhmann, N. (2008). Familiarity, Confidence, Trust: Problems and Alternatives; en, D. Gambetta (Ed.), Trust: making and breaking cooperative relations (pp. $94-108)$. EUA: Basil Black Well, Oxford.

Manasse, M. \& Ganem, N. (2009). Victimization as a cause of delinquency: The role of depression and gender. Journal of Criminal Justice, 37, 371 - 378.

March, J. \& Olsen, J. (1984). The new Institutionalism: Organizational factors in political life. The American Political Science Review, 78 (3), 734 - 749.

Merton, R. (1968). Social Theory and Social Structure. NY, EUA: Free Press.

Merton, R (1995). Opportunity Structure: The emergence, Diffusion, and Differentiation of a Sociological Concept; en, F. Adler \& W. S. Laufer (Eds.), The Legacy of Anomie Theory (pp. 3 - 78). Nueva Jersey, EUA: Transaction Publisher.

Mihaiu, D., Opreaana, A. \& Cristescu, M. (2010). Efficiency Effectiveness and Performance of the Public Sector. Romanian Journal of Economic Forecasting, 4.

Misztal, B. (1996). Trust in Modern Societies: The search for the bases of social order. EUA: Polity Press \& Black Well Publisher ltd.

Mosher, C. Miethe, T. \& Phillips, D. (2002). The mismeasure of crime. EUA: SAGE Publications.

Mutimer, D. (2013). Critical Security Studies. En A. Collins (Ed.), Contemporary Security Studies (pp. 67-87). Reino Unido: Oxford University Press.

Nelson, R. \& Winter, S. (2002). Evolutionary Theorizing in Economics. Journal of Economics Perspectives, 16(2), $23-47$.

Nooteboom, B. (2010). La dinámica de la confianza: comunicación, acción y terceras partes. Revista de economía institucional, 12(23).

North, D. (1990). Instituciones, cambio institucional y desempeño económico. México: Fondo de Cultura Económica.

North, D. (2005). Understanding the Process of Economic Change. Princeton, EUA: Princeton University Press.

Olsen, R. (2008). Trust and risk and the foundation of investment value. The Journal of SocioEconomics, 37(6), 2189-2200.

Ostrom, E. (1990). Governing the Commons: The evolution of institutions for collective actions. Nueva York, EUA: Cambridge University Press.

Ostrom, E. \& Anh, T. K. (2007). The meaning of social capital and its link to collective action. Recuperado de http://ostromworkshop.indiana.edu/publications/materials/W07-19.pdf $<$ última fecha de consulta, 3 de noviembre de 2016> 
Ozcan, B. \& Bjornskov, C. (2011). Social trust and human development. The Journal of SocioEconomics, 40(6), 753-762.

Padmanabhan, M. (2008). Collective Action in Agrobiodiversity Management: Gendered Rules of Reputation, Trust and Reciprocity in Kerala, India. Journal of Integrational Development, $20,83-97$.

Paldam, M. (2007). Generalized Trust: The macro perspective. Manuscrito inédito, School of Economics and Management, University of Aarhus, Dinamarca.

Paul, D. \& McDaniel Jr., R. (2004). A Field Study of the Effect of Interpersonal Trust on Virtual Collaborative Relationship Performance. Mis Quarterly, 28(2), 183 -227.

Paxton, P. (1999). Is Social Capital Declining in the United States? A Multiple Indicator Assessment. AJS, 105, 99- 127.

Pierson, P. (2000). Not Just What, but When: Timing and Sequence in Political Processes. Studies in American Political Development, 14, 72 -92.

Programa de las Naciones Unidas para el Desarrollo (PNUD) (2014). Reducir Vulnerabilidades y Construir Resiliencia. Recuperado de http:/hdr.undp.org/sites/default/files/hdr14summary-es.pdf <última fecha de consulta, 3 de noviembre de 2016>

Putnam, R. (1993). Making Democracy Work: Civic Traditions in Modern Italy. Nueva Jersey, EUA: Princeton University Press.

Ramírez, D. (2008). Crimen y castigo, víctima y delito ¿Un problema de demanda? Temas de coyuntura, $57,121-148$.

Robert Jr., L. Dennis, A. \& Hung, Y. (2009). Individual Swift Trust and Knowledge - Based Trust in Face to Face and Virtual Team Members. Journal of Management Information Systems, 26(2), $241-279$.

Rock, P. (2012). Sociological Theories of Crime. En M. Maguire, M. Morgan, \& R. Reiner (Eds.), Oxford Handbook of Criminology (pp. 39-81). (5 Ed.). Oxford, EUA: Oxford University Press.

Rosenfeld, R. Messner, S. \& Baumer, E. (2001). Social Capital and Homicide. Social Forces, 80(1), $283-309$.

Ruscio, K. (1996). Trust, Democracy, and Public Management: A Theoretical Argument. Journal of Public Administration Research and Theory: J-PART, 6(3), 461-477.

Rutherford, M. (1994). Institutions in Economics: The Old and New Institutionalism. New York, EUA: Cambridge University Press.

Sampson, R.J. (2001). Crime and public safety: Insights from community-level perspective on social capital; en, S. Saegert, P.J. Thompson \& M. Warren (Eds.), Social capital and poor communities: Building and using social capital assets to combat poverty (pp. 89 - 115). New York, EUA: Russell Sage.

Scharpf, F. (1997). Games Real Actors Play: Actor-Centered Institutionalism in Policy Research. EUA: Boulder and Westview Press.

Schroeder, R., Hill, T., Haynes, S. \& Bradley, C. (2011). Physical health and crime among lowincome urban women: An application of general strain theory. Journal of Criminal Justice, $39,21-29$. 